\title{
Pré-natal de Risco Habitual e Alto Risco: Estudo Qualitativo sobre Percepções de Mulheres
}

\author{
Marcia Regina Cangiani Fabbro ${ }^{1}$, Jamile Claro de Castro Bussadori ${ }^{1}$, \\ Monika Wernet ${ }^{1}$, Bruna Felisberto de Souza ${ }^{2}$, Andrea dos Reis \\ Fermiano $^{2}$ e Maria Aparecida Gracélia Sousa ${ }^{3}$
}

\author{
${ }^{1}$ Departamento de Enfermagem. Programa de Pós-Gradução em Enfermagem. \\ Universidade Federal de São Carlos, Brasil | mfabbbro@gmail.com; \\ jamilebussadori@gmail.com; monika.wernet@gmail.com | https://orcid.org/0000-0003-2601 - \\ 8818; https://orcid.org/0000-0002-3048-5593; https://orcid.org/0000-0002-1194-3261 \\ 2 Doutoranda do Programa de Pós-Graduação em Enfermagem. Universidade Federal de \\ São Carlos, Brasil | souza.brunaf@gmail.com; fermiand@yahoo.com.br | \\ https://orcid.org/0000-0003-1858-8896; https://orcid.org/0000-0003-0279-0635 \\ ${ }^{3}$ Mestre em Enfermagem pelo Programa de Pós-Gradução em Enfermagem. Universidade \\ Federal de São Carlos, Brasil· | gracelia-sousa@hotmail.com | https://orcid.org/0000-0002-
} 9724-6538

Resumo: A avaliação de um serviço de pré-natal sob a ótica de gestantes é um poderoso instrumento que possibilita adensar conhecimentos e contribuir com transformações nas práticas de saúde, qualificando-as. Objetivo: Analisar percepções de mulheres, gestantes de risco habitual e puérperas que gestaram sob o diagnóstico de alto risco, acerca da atenção pré-natal recebida. Método: Estudo qualitativo, desenvolvido em 2018 e 2019, junto a 30 gestantes de risco habitual e 7 puérperas de alto risco inseridas na atenção pré-natal de cidade do interior paulista. A obtenção dos dados ocorreu a partir de entrevista semiestruturada em encontro único. Os referenciais do Interacionismo Simbólico, quadro conceitual de experiência positiva na gestação e Análise Temática sustentaram o estudo. Resultados: O pré-natal foi vivenciado sob um contexto interacional pouco acolhedor e até desrespeitoso, o que imprimiu limitações à autonomia para questões da gestação, parto e nascimento e para o estabelecimento do apoio social, o que dificultou o empoderamento pessoal das mulheres para enfrentar as situações adversas da gestação e parto. Duas categorias temáticas detalham os achados: "Interações com profissionais", "Entraves no apoio informacional para gestação, parto e nascimento". Conclusão: A atenção pré-natal esteve descrita e centrada em clínica biomédica, com insuficiências relacionais entre profissionais e mulheres e de pouco alcance às singularidades, em especial no âmbito informacional, com escassez de abordagem do parto no pré-natal. Infere-se que a pesquisa qualitativa, sobretudo a estratégia de entrevista, imprime um meio de valorar o diálogo horizontal e é recurso que promove interações subjetivas genuínas.

Palavras-chave: Cuidado Pré-natal; Gestantes; Atenção Primária à Saúde; Atenção Secundária à Saúde; Lacunas da Prática Profissional.

\section{Habitual Risk and High Risk Prenatal: A Qualitative Study on Women's Perceptions}

\begin{abstract}
The evaluation of a prenatal service from the point of view of pregnant women is a powerful tool that allows to increase knowledge and contribute to changes in health practices, qualifying them. Objective: To analyze the perceptions of women, pregnant women of habitual risk and high-risk mothers about prenatal care. Method: Qualitative study, developed in 2018 and 2019, with 30 pregnant women at usual risk and 7 high-risk puerperal women inserted in prenatal care in a city in the interior of São Paulo. Data were obtained from a semi-structured interview in a single meeting. The references of Symbolic Interactionism, conceptual framework of positive experience in pregnancy and Thematic Analysis supported the study. Results: Prenatal care was experienced under an unwelcoming and even disrespectful interactional context, which imposed limitations on autonomy for issues of pregnancy, childbirth and birth and for the establishment of social support, which hindered the personal empowerment of women to face adverse situations of pregnancy and childbirth. Two thematic categories detail the findings: "Interactions with professionals", "Barriers to information support for pregnancy, childbirth and birth". Conclusion: Prenatal care was described and centered on a biomedical clinic, with relational insufficiencies between professionals and women and with little scope for singularities, especially in the informational scope, with a lack of approach to prenatal delivery. It is inferred that qualitative research, especially the interview strategy, provides a means of valuing horizontal dialogue and is a resource that promotes genuine subjective interactions.
\end{abstract}

Keywords: Prenatal Care; Pregnant Women; Primary Health Care; Secondary Care; Professional Practice Gaps. 


\section{Introdução}

O Pré-natal (PN) no Brasil é desenvolvido pela Atenção Primária em Saúde (APS) quando de risco habitual e é ação programática da Atenção Especializada ( $A E$ ), quando de alto risco, ambos intencionam alcances nos indicadores da morbimortalidade materna e infantil (Ministério da Saúde, 2012; Mascarenhas et al., 2017) e também uma experiência positiva às mulheres (Ministério da Saúde, 2012; World Health Organization, 2016).

Segundo Costa et al. (2005) a atenção pré-natal visa estabelecer um processo de vigilância à saúde das gestantes, com o propósito de controlar eventuais riscos. Para tanto, necessita garantir avaliação contínua de riscos obstétricos e proporcionar recurso ambulatorial e hospitalar adequado ao atendimento desses riscos. As situações que envolvem fatores clínicos mais relevantes (risco real) e/ou fatores evitáveis que demandem intervenções com maior densidade tecnológica devem ser referenciadas para um acompanhamento em pré-natal de alto risco, podendo, contudo, retornar ao nível primário, quando se considerar a situação resolvida e/ou a intervenção já realizada. De qualquer maneira, a unidade básica de saúde deve ser responsável pelo seguimento da gestante encaminhada a serviço especializado, mantendo a atenção básica como porta de entrada da Rede de Atenção à Saúde e ordenadora do sistema de saúde brasileiro (Ministério da Saúde, 2012).

No Brasil, houve ampliação do acesso e da cobertura da atenção PN (Tomasi et al., 2017; Luz et al., 2018), contudo não vem se traduzindo em qualidade (Tomasi et al., 2017), mantendo insuficiências em termos de acolhimento e apoio integral às gestantes (Silva et al., 2019).

Embora as experiências das mulheres no período perinatal sejam influenciadas pelas suas crenças, valores e expectativas (Vogels-Broke et al., 2020; Benincasa et al., 2019), ter uma experiência positiva com o PN possui relação direta com as experiências interacionais junto aos profissionais de saúde e gera impacto na forma como se percebe a qualidade do cuidado dispensado (Gaiva et al., 2017).

Assim, independentemente do risco gestacional, o modelo de cuidado deve visar uma experiência positiva, elencando a mulher como elemento central do processo e incorporando, além do bem-estar físico, as dimensões socioculturais e emocionais. É necessário considerar, para além da sobrevivência das mulheres e bebês, que os mesmos prosperem e alcancem todo o seu potencial para a saúde e a vida, dentro de uma atenção clínica e emocionalmente segura (World Health Organization, 2016; World Health Organization, 2018). Acessar a perspectiva de mulheres acerca do PN é possibilidade para adensar conhecimentos e contribuir com transformações nas práticas de saúde, qualificando-as (Andrade et al., 2019).

Desse modo, a qualificação da atenção em saúde é investimento prioritário (Costa et al., 2016). Considerando o pré-natal a etapa inicial da implementação de uma experiência positiva, além de desempenhar papel de rastreio, diagnóstico e prevenção de doenças durante a gestação, a assistência pré-natal deve proporcionar a educação em saúde. Mulheres informadas e apoiadas sobre as vivências implicadas no gestar, parir e cuidar, e em especial, sobre suas situações de risco, são mais preparadas para assumir o protagonismo no parto e na transição para uma maternidade saudável (Souza et al., 2020).

Frente ao exposto, este estudo tomou como pergunta "Como mulheres que estão a vivenciar o PN na APS e na AE o percebem? O que apontam para sua qualificação?". O objetivo desta pesquisa foi analisar as percepções de gestantes de risco habitual e de puérperas que gestaram sob o diagnóstico de alto risco, acerca da atenção pré-natal. 


\section{Metodologia}

Trata-se de uma pesquisa qualitativa, descritiva e exploratória voltado à experiência de mulheres com o pré-natal de risco habitual e o pré-natal de alto risco. O estudo apoia-se no referencial teórico do Interacionismo Simbólico (Charon, 2009) por ser sensível ao que é vivido nas interações sociais e as consequentes (re)interpretações simbólicas derivadas delas e, pelo quadro conceitual da experiência positiva na gestação (Who, 2016), cujas interações e intervenções devem ocorrer com base em evidências científicas e cuidado individualizado na figura da mulher.

Este estudo ocorreu em um município do interior do estado de São Paulo, Brasil, cuja atenção pré-natal de risco habitual é integralmente realizada na APS e a gestação de alto risco conduzida em AE. Foi desenvolvido nos anos de 2018 e 2019 e integra dois estudos maiores de doutoramento, intitulados "Alcances de Programa de Visitação no pré-natal de alto risco" e "Avaliação do pré-natal segundo indicadores de saúde, gestantes, puérperas e profissionais" que estão em curso simultaneamente desde 2018 e aprovados em Comitê de Ética sob parecer números 2.467 .7332 e 2.761.178, respectivamente.

As participantes foram gestantes e puérperas que atendiam aos seguintes critérios de inclusão: ser maior de 18 anos, estar em acompanhamento e/ou ter realizado acompanhamento PN na APS ou Serviço Especializado e, quando em acompanhamento, ter vivenciado pelo menos três consultas de PN. Como de exclusão: não conseguir emitir uma narrativa compreensível ao convite para a entrevista.

Para a coleta de dados optou-se por realizar entrevista semiestruturada com as mulheres disparadas pelas questões: 'Como vem percebendo a atenção pré-natal recebida?', 'Destaque algum aspecto que tem gostado e ficado satisfeita.'; 'O que pensa que pode ser melhorado e por quê?' 'Como foi sua experiência de pré-natal?'. Outras perguntas vieram a ser apresentadas na direção de compreensão do exposto pelas participantes. Ademais, um questionário sociodemográfico e de antecedentes obstétricos, elaborado pelas próprias autoras, foi aplicado a fim de caracterizar as mulheres.

As entrevistas com as gestantes de risco habitual foram conduzidas pela penúltima autora e desenvolvidas nas Unidades Básicas de Saúde e Unidades de Saúde da família. Ao ingressarem nas unidades de saúde para realização de consulta PN, a pesquisadora conduzia às mulheres a uma sala reservada, apresentava as estratégias e objetivos da presente pesquisa e posteriormente, fazia o convite para ingressarem no estudo. As entrevistas com as puérperas que gestaram sob alto risco foram realizadas no domicílio das mesmas. Tais puérperas integravam estudo de visitação domiciliar supracitado e aceitaram colaborar com a entrevista sobre as percepções do pré-natal de alto risco recebido, conduzida pela última autora deste artigo. Nas instituições de saúde ou no domicílio das mulheres, os objetivos do estudo eram retomados com posterior certificação do interesse em colaborar com a pesquisa. A leitura conjunta do Termo de Consentimento Livre e Esclarecido era desenvolvida e após sua assinatura, a entrevista tinha seu início. Todas as entrevistas foram gravadas em áudio e, posteriormente transcritas na íntegra. A duração média de entrevistas com gestantes de risco habitual foi de 25 minutos e, de 50 minutos com puérperas de alto risco e não houve retorno das análises efetuadas às gestantes e puérperas.

Um total de trinta mulheres que realizavam o pré-natal de risco habitual foram entrevistadas. Por volta da $20^{\underline{a}}$ entrevista os dados já assinalavam saturação por significado, isto é, o conjunto de dados asseguravam elementos suficientes em densidade e recorrência acerca do fenômeno em exploração (Hennink, Kaiser \& Marconi, 2017), contudo, mesmo a saturação ter se mostrado aparente, as pesquisadoras decidiram por manter o processo de entrevista, não excluindo os demais depoimentos. Com relação às puérperas de alto risco, foram realizadas nove entrevistas, sendo uma excluída por narrativa incompreensível, e a outra por desistência, totalizando sete mulheres. 
A participação dessas mulheres estava regulada à sua entrada no projeto de visitação domiciliar, não sendo possível alcançar um quantitativo mais expressivo, fato que não comprometeu a qualidade dos depoimentos sobre o pré-natal de alto risco.

A análise, interpretações e organização dos depoimentos foram conduzidas por meio do referencial metodológico da análise temática de Bardin (Bardin, 2014). Tal técnica dispõe de três etapas em seu processo analítico: (1) Pré-análise: com objetivo de organizar o material, pela "leitura flutuante" das entrevistas transcritas para identificar as impressões iniciais; (2) Exploração do material: por meio da codificação do conteúdo por unidade de registro e unidade de contexto, com intuito de categorizar os dados; (3) Tratamento dos resultados obtidos e interpretação: procedimento em que são agrupados os conteúdos e comparados para identificar semelhanças ou divergências entre as categorias existentes.

$\mathrm{Na}$ intenção do anonimato, os excertos foram identificados pela letra 'G', quando gestantes, seguida por um algarismo arábico em ordem crescente, retratando a entrada no estudo, além da idade e paridade da participante. $E$, quando puérperas, os excertos foram identificados pela letra ' $P$ ', seguida por um algarismo arábico em ordem crescente, retratando a entrada no estudo.

\section{Resultados e Discussões}

Foram entrevistadas trinta (30) gestantes de risco habitual, cujo perfil foi: $20(67 \%)$ tinham idade entre 18 e 25 anos, 7 (23\%) entre 26 e 30 anos e $3(10 \%)$ entre 31 e 35 anos. A gravidez não foi planejada para $21(70 \%)$ e planejada para $9(30 \%)$. Era a primeira gestação para 13 mulheres (43\%) e $17(57 \%)$ já tinham pelo menos, um filho. Possuíam vida conjugal $12(40 \%)$ delas, $14(47 \%)$ estavam solteiras e duas $(6 \%)$ não quiseram informar. Quanto a profissão ou ocupação, 18 (60\%) estavam exercendo algum tipo de atividade remunerada, 10 (33\%) estavam desempregadas ou sem ocupação e não obtivemos os dados de $2(7 \%)$ que não quiseram identificar. Quanto à escolaridade, $19(63 \%)$ frequentaram até o ensino médio, $5(17 \%)$ até o ensino fundamental e 6 (20\%) não quiseram identificar. A maioria se declarou branca.

Foram entrevistadas sete (7) puérperas de alto risco, cujo perfil foi: média de idade de 31 anos, variando de 24 a 38 anos; $42,8 \%$ se autodeclaram negras, $28,6 \%$ pardas e $28,6 \%$ brancas e, a maioria referiu ter relação conjugal oficializada (85,7\%). Referente à escolaridade, todas tinham no mínimo o ensino fundamental, sendo que $28,6 \%$ tinham ensino médio completo, $14,2 \%$ ensino superior incompleto, e $14,2 \%$ pós-graduação. Somente $28,6 \%$ das participantes tinham vínculo empregatício, o restante era do lar. A maioria $(86,7 \%)$ já havia passado por outra gestação, a hipertensão gestacional foi a patologia mais incidente na gestação referente $(57,2 \%)$ e a via de nascimento a cesariana $(71,4 \%)$.

Duas categorias temáticas emergiram após análise das entrevistas, são elas: "Interações com profissionais", "Entraves no apoio informacional para gestação, parto e nascimento" e representam os aspectos que compõe as percepções e apontamentos das mulheres acerca do PN.

\subsection{Interações com Profissionais}

As narrativas revelaram percepções de ser o PN significado de uma consulta protocolar com o profissional médico. Reconheceram que o (des)acolhimento é estruturado a partir dos procedimentos técnicos para avaliar a condição fisiológica da gestação, citam aferição de pressão arterial, peso e prescrição de exames nesse cuidado. A partir das percepções, o pré-natal é descrito enquanto consultas rápidas, de pouco espaço e disponibilidade dos profissionais. 
Você passa antes com as enfermeiras, elas marcam a sua pressão e peso. Quando não tem exame e nem ultrassom, ele (médico) marca o seu tempo de gestação e escuta o coração. Se ele quiser pedir algum exame ou outro ultrassom, ele pede, se não a consulta é isso e pronto, você não fica nem meia hora ali dentro, fica uns 10 minutos. Toda vez quase igual (G04, 30 anos, terceira gestação).

As consultas sempre foram assim, aferia a pressão, esperava a chamada da médica, mostrava os exames e marcava o retorno e pronto. Só isso e mais nada. Às vezes ela perguntava se tinha alguma dúvida, mas às vezes nem perguntava nada ( $P 2)$.

As mulheres têm necessidade e desejo de serem ouvidas. $O$ acolhimento, desde 0 momento em que chegam na unidade se faz necessário, porém aquém do ideal (Pereira et al., 2018). A presente pesquisa confirma tal apontamento, quando uma das mulheres compara o tratamento recebido em seu país natal com o tratamento recebido no serviço especializado de alto risco. Reconhece que um dos pontos a ser melhorado é o diálogo e a escuta.

Outras apreciações menos positivas do PN estiveram ancoradas em relações permeadas por comportamentos percebidos como de desinteresse e desrespeito por parte dos profissionais. Em ambas as situações, infere-se que as mulheres percebem e significam o PN enquanto superficial e a vinda nas consultas foi de pouca motivação e quase que sustentada pela obrigação de vir. No contexto de uma atenção voltada aos aspectos instrumentais, o parto, conforme relato das mulheres, não foi abordado nas consultas de pré-natal.

Eu achei muito rápido, muito pontual, minhas experiências são da Venezuela, e lá os ginecologistas são como uns papais, conversam bastante, aqui eles quase não conversam vão direto ao ponto, poderiam melhorar (P7).

Eu acho uma falta de responsabilidade da parte dela (médica) porque está marcado para o horário certo lá, sempre demora, a gente tem outras coisas para fazer e tem que deixar de fazer para poder esperar a vontade dela chegar e atender os pacientes, então quando ela atende a gente é coisa de segundos. (G05, 18 anos, primeira gestação)

Não, nenhuma dica se eu tivesse que fazer uma cesárea ou se eu fosse fazer um parto normal, nada. Nessa parte não falavam nada (P5).

É premente investir na gestão do cuidado, na gestão de saúde com vistas a satisfação das gestantes, com alcances na adesão às consultas (Santos \& Mamede, 2018). Diante disto, as denúncias deste estudo acerca do tempo de espera para serem atendidas é crítico e desrespeitoso.

Um atitudinal profissional voltado ao acolhimento, paciência, atenção e cuidado são significadas como uma necessidade para que a experiência seja positiva, principalmente diante dos sentimentos negativos que vêm à tona desse viver (Benincasa et al., 2019; Zanardo et al., 2017). Contudo, ainda que signifiquem e percebam as lacunas assistenciais da atenção pré-natal, as mulheres desse estudo também classificaram a experiência do cuidado com a presença de ações que denotaram avaliação da criança, com valoração diferenciada aos exames e à ausculta dos batimentos cardiofetais.

(...) ah eu gosto de vir para escutar o coraçãozinho dela (criança), ver se está tudo bem, então o que é legal é isso, não tem outra coisa (risos) (G14, 25 anos, quarta gestação).

(PN envolve) muitos exames, ela (médica) pede exame toda vez que eu venho. Gosto dos exames, do ultrassom. (G05, 18 anos, primeira gestação). 
$\mathrm{Na}$ assistência à gestação, sobretudo a gestação de alto risco, os sinais vitais e o exame físico são fundamentais para o seguimento, pois configuram-se indicadores de alterações importantes. No entanto, diante dos relatos, percebe-se que esse cuidado se sobressai aos cuidados subjetivos, é a técnica em detrimento da experiência (Souza et al., 2020).

Ancorado nessas ideias e nos relatos, percebe-se a falta de escuta qualificada e interações com os profissionais. A partir deste entendimento, uma ação cotidiana é oferecer uma escuta sensível e perguntar sobre a existência de dúvidas e necessidades e, diante delas, ofertar a informação necessária para esclarecimento das mesmas, dando autonomia a essas mulheres para vivenciar a gestação em sua plenitude, com valoração das histórias e individualidades de cada mulher (Souza et al., 2020; Silva et al., 2016).

\subsection{Entraves no Apoio Informacional para Gestação, Parto e Nascimento}

As participantes intencionam obtenção de informações no PN, quando apontaram como evento determinante o desconforto na relação com o profissional. Nesse contexto, símbolos interpretados como afirmação da superioridade fizeram com que elas se sentissem desencorajadas e inseguras para apresentar dúvidas ou necessidades. Interpretar símbolos na atitude do profissional enquanto descaso e indiferença desdobravam-se em sentimentos de inferioridade e constrangimento, com consequente recolhimento na ação de perguntar/interagir. Ainda, a historicidade de relação truncada com o profissional e/ou serviço afetou negativamente seu modo de ser e estar no PN e limitou sua manifestação autêntica, sobretudo no âmbito da necessidade informacional. Quando o tema parto foi abordado, muitas vezes as primeiras tentativas de dialogo ocorriam por iniciativa da mulher.

(...) às vezes ela (médica) pergunta se eu tenho medo de alguma coisa e aí quando você não entende e faz alguma pergunta ela ficava assim (careta, longa pausa) e começa a explicar, parece que ela fala "você é burra hein". Não perguntei mais (G06, 24 anos, primeira gestação).

(...) às vezes ela é meia seca, ela te dá umas, umas carcadinhas assim, sem querer. Eu nem perguntei mais (G17, 28 anos, segunda gestação).

(risos) estou passando porque eu tenho que passar, ele (médico) é muito estúpido (...) É sempre assim, difícil. Daí a gente só ouve, fica com as dúvidas (G21, 20 anos, primeira gestação).

Eu só fui falar de parto na última consulta com ela. Quando me consultei pela primeira vez, eu já queria esclarecer algumas dúvidas, mas ela (referindo-se a médica) falou assim "não, ainda não chegou a hora de falar sobre isso, isso daí só lá para frente" (P5).

A pesquisa 'Nascer no Brasil' já revelou que persistem desafios para a qualidade do PN. No que se refere a informação sobre tipos de parto, por exemplo, os dados mostram baixa preferência pelo parto vaginal, sugerindo pouca importância desta informação para o processo de decisão das mulheres brasileiras (Domingues et al., 2014). A necessidade de informação existe e, diante do contexto interacional fragilizado junto ao profissional, recorreram a sua mãe e à internet, mas nem sempre sanam suas questões. Ao tentarem resolvê-las junto ao médico são recriminadas, quando relatam o uso da internet. 
Tem vez que eu pergunto para o médico e tem vez que eu pesquiso na internet (risos) mas aí eu fico mais doida (risos) eu falo para ele (médico) que eu pesquisei, aí ele fala para eu sair da internet (G22, 19 anos, primeira gestação).

Eu não pergunto para ninguém, eu fico mais na internet mesmo, vendo os vídeos, vendo as coisas, ainda não cheguei a perguntar para o médico (G19, 20 anos, primeira gestação).

De dúvida geralmente é mais para minha mãe e pesquiso bastante na internet, a semana da gestação pesquiso no YouTube, o que é bom para eu comer, para estar se alimentando, porque não dá para conversar com o médico, ele só examina só e já tchau (G21, 20 anos, primeira gestação).

Não é uma atitude acolhedora desconsiderar o uso internet e a obtenção de informações nas interações sociais em família. A internet é parte da vida moderna e deve ser incorporada às práticas de saúde e não substituí-las, para tanto, o profissional precisa transformar sua postura a fim de conciliar o uso dessa nova ferramenta, avaliando conteúdos online e integrando eles ao seu fazer (Lobo, 2018), bem como auxiliando as mulheres a localizarem as informações baseadas nas evidências científicas.

Questões acerca do parto são necessidades informativas recorrentes, quando em interações sociais distintas (como a sala de espera explicitada no enxerto abaixo) prevaleceram apontamentos de reprodução social do modelo de atenção obstétrica intervencionista como o seguro, de menor risco, em contraponto ao parto normal. Diante da insuficiência na abordagem desta temática, as mulheres discorreram e teceram recomendações sobre a necessidade de mais informação como estratégia ideal para a mudança neste cenário.

Eu conversei agora com ele (médico), eu vou fazer cesárea, por mais ouvir falar, tem pessoas que já tiveram recentemente nenê na família, que sofre muito, corre risco, que eles seguram até o último pra soltar a cesárea, e você ouve falar também né, de nenê que já morreu, então você acaba ficando com medo de esperar até o último dia da gestação (G02, 27 anos, primeira gestação).

(...) não tenho intenção de fazer parto normal, ah, eu tenho medo, ai, tem comentários, diz que é sofrido demais, pesquisei na internet e eu acho que é muito sofrido (G27, 22 anos, segunda gestação).

Aproveito a espera da consulta para perguntar para outras lá coisas que quero saber e que sei que não dá para perguntar para ro médico. É tudo rápido. Daí falo para outras pessoas, porque talvez elas saibam (G30, 30 anos, segunda gestação).

(...) mais informação, porque a gente tem muitas dúvidas e eles não conversam muito sobre isso" (P2).

Mais preparação. É importante você explicar os tipos de parto, para ficar preparada para os dois, porque não adianta você escolher um, porque chega lá na hora pode ser que não role (P3).

As evidências científicas destacam que quando se priorizam intervenções educativas como estratégias que incentivam a intersubjetividade é possível promover abertura para um diálogo. Nesta direção, espaços coletivos e individuais de educação, sustentados no diálogo, são estratégicos. Buscar um diálogo igualitário e emancipador envolve uso apropriado da linguagem, atento ao espaço da interação verbal, mas também da interação não verbal (Gárcia-Carrión et al., 2020). O diálogo, quando estabelecido numa base igualitária, coloca todos envolvidos, independentemente da origem, status ou estudos, ao mesmo nível, uma vez que a força das reivindicações baseia-se na validade dos argumentos que se apoiam, em vez de em retóricas ou relações de poder (TorrasGómez et al., 2020). 


\section{Conclusões}

Os atributos que determinaram representar a experiência do pré-natal enquanto positiva ou não, estiveram conectadas, neste estudo, sobretudo ao modelo de cuidado recebido durante o momento. Quando um cuidado humanizado e diretamente relacionado às boas práticas em saúde é valorizado, uma experiência exitosa no viver a gestação, parto e nascimento fica evidenciada.

Constatou-se que, tanto o PN de risco habitual quanto o de alto risco se expressam a partir de experiências interacionais superficiais, com "encontros" esvaziados e escassos esforços ao acolhimento. As mulheres revelam sentimentos de menosprezo, interações hierarquizadas, antidialógicas e, por vezes, desrespeitosas. Tais experiências possuem desdobramentos negativos à qualidade e, sobretudo, aos possíveis alcances do $\mathrm{PN}$, uma vez que às mulheres foi ofertado pouco poder de fala e pouca aposta na autonomia. Ademais, a educação em saúde foi insuficiente, inclusive no que tange as discussões sobre vias de parto.

Como fatores de contribuição, este estudo aponta três balizadores para um PN de maior qualidade: ampliar o escopo da clínica, investir nas relações igualitárias e garantir apoio informacional. Eles estão imbricados e determinam mudanças atitudinais do profissional e da forma como a gestão do cuidado é conduzida. A sugestão aqui colocada é de apostar no diálogo igualitário e na escuta genuína, para que seja possível pensar criticamente no modelo de saúde obstétrico vigente no Brasil e discuti-lo, de forma crítico-reflexiva, visando os alcances do PN em sua integralidade.

Infere-se que a oferta de um espaço de escuta qualificada, por meio de entrevistas, como no método qualitativo, valoram relações intersubjetivas e podem ter operado como dispositivos para que, algumas destas mulheres pudessem se ouvir e, com isso, abrir a possibilidade de um questionamento sobre si, através do recurso da palavra.

O método qualitativo poderia ainda contribuir na compreensão da dimensão subjetiva do trabalho em saúde, ao reconhecer a contribuição singular de cada profissional de saúde. Neste sentido, a formação em saúde implica considerar que os processos de aprendizagem não se dão somente pelas dimensões cognitivas, mas também por uma elaboração psíquica apoiada nas vivências subjetivas dos profissionais. Assim, recomenda-se maior exploração dessa metodologia para o contexto do pré-natal.

\section{Referências}

Andrade, U. V., Santos, J. B., \& Duarte, C. (2019). Pregnant women perception of the quality of prenatal care at UBS, Campo Grande, MS. Psicologia \& Saúde, 11(1), 53-61. https://dx.doi.org/10.20435/pssa.v0i0.585

Bardin, L. (2014). Análise do conteúdo. Lisboa: Editora 70.

Benincasa, M., Navarro, A., Bettiol, N. L. S, \& Heleno, M. G. (2019). Percepções de mulheres sobre o momento do parto e a assistência obstétrica recebida. Cadernos de Gênero e Diversidade, 5(4), 63-88. http://dx.doi.org/10.9771/cgd.v5i4.29172

Ministério da Saúde. (2012). Atenção ao pré-natal de baixo risco. (1st ed.). http://bvsms.saude.gov.br/bvs/publicacoes/cadernos_atencao_basica_32_prenatal.pdf

Costa, L. D., Cura, C. C., Perondi, A. R., França, V. F., \& Bortoloti, D. S. (2016). Perfil epidemiológico de gestantes de alto risco. Cogitare Enfermagem, 21(2), 1-8. http://dx.doi.org/10.5380/ce.v21i2dd192

Costa, A. M., Guilhem, D., \& Walter, M. I. M. T. (2005). Atendimento a gestantes no Sistema Único de Saúde. Revista de Saúde Pública, 39(5), 768-774. https://doi.org/10.1590/S003489102005000500011

Charon, J. M. (2009). Symbolic Interacionism: an Introduction, an Interpretation, an Integration. Pearson.

Domingues, R. M. S. M., Dias, M. A. B., Nakamura-Pereira, M., Torres, J. A., d'Orsi, E., Pereira, A. P. E., Schilithz, A. O. C., \& Leal, M. C. (2014). Process of decision-making regarding the mode of birth in Brazil: from the initial preference of women to the final mode of birth. Cadernos de Saúde Pública, 30 (Suppl. 1), S101-S116. https://doi.org/10.1590/0102-311X00105113 
Gaíva, M. A. M., Palmeira, E. W. M., \& Mufato, L. F. (2017) Women's perception of prenatal and delivery care in cases of neonatal death. Escola Anna Nery, 21(4), e20170018. https://doi.org/10.1590/2177-9465-ean-2017-0018

García-Carrión, R., Villardón-Gallego, L., Martínez-de-la-Hidalga, Z., \& Marauri, J. (2020) Exploring the Impact of Dialogic Literacy Gatherings on Student's Relationships With a Comunicative Approach. Qualitative Inquiry, 26 (8-9), 996-1002. https://doi.org/10.1177/1077800420938879.

Hennink, M. M., Kaiser B. N., \& Marconi, V. C. (2017). Code saturation versus meaning saturation: how many interviews are enough? Qualitative Health Research, 27 (4), 591-608. https://doi.org/10.1177/1049732316665344

Lobo, L. C. (2018). Artificial Intelligence, the Future of Medicine and Medical Education. Revista Brasileira de Educação Médica, 42 (3), 3-8. https://doi.org/10.1590/198152712015v42n3rb20180115editorial1

Luz, L. A., Aquino, R., \& Medina, M. G. (2018). Evaluation of the quality of Prenatal Care in Brazil. Saúde debate, 42 (n. esp 2). https://doi.org/10.1590/0103-11042018S208

Mascarenhas, P. M., Silva, G. R., Reis, T. T., Casotti, C. A., \& Nery, A. A. (2017). Analysis of maternal mortality. Enfermagem UFPE on line, 11(suppl 11), 4653-4662. https://doi.org/10.5205/19818963-v11i4a15257p1616-1624-2017

Pereira, A. G., Silva, F. O, Brasil, G. B., Rodrigues, I. L. A., \& Nogueira, L. M. V. (2018) Perceptions of riverine pregnant women on prenatal care. Cogitare Enfermagem, 23 (4), e54422. http://dx.doi.org/10.5380/ce.v23i4.54422

Santos, P. P., \& Mamede, F. V. (2018). Evaluation of prenatal care in primary care in the perception of pregnant women. Revista Gaúcha de Enfermagem, 39, e20180077. http://dx.doi.org/10.1590/1983-1447.2018.20180077

Silva, D. V., Silveira, M. D., \& Gomes-Sponholz, F. A. (2016). Experiences with severe maternal morbidity: a qualitative study on the perception of women. Revista Brasileira de Enfermagem, 69 (4), 662-668. http://dx.doi.org/10.1590/0034-7167.2016690407i

Silva, A. A., Jardim, M. J. A., Rios, C. T. F., Fonseca, L. M. B., \& Coimbra, L. C. (2019). Prenatal care of risk pregnant women: potentialities and weaknesses. Revista de Enfermagem da UFSM, 9 (e15), 1-19. https://doi.org/10.5902/2179769232336

Souza, B. F., Bussadori, J. C. C., Ayres J. R. C. M., Fabbro M. R, \& Wernet, M. (2020). Nursing and hospitalized high-risk pregnant women: challenges for comprehensive care. Revista Escola de Enfermagem da USP, 54, e03557. https://doi.org/10.1590/s1980-220x2018036903557

Tomasi, E., Fernandes, P. A. A., Fisher, T., Siqueira, F. C. V., Silveira, D. S., Thumé, E., \& Facchini, L. A (2017). Quality of prenatal services in primary healthcare in Brazil: indicators and social inequalities. Cadernos de Saúde Pública, 33 (3), e00195815. https://doi.org/10.1590/0102 $311 \times 00195815$

Torras-Gómes, E., Guo, M., \& Ramis, M. (2020). Sociological Theory from Dialogic Democracy. International and Multidisciplinary Journal of Social Sciences, 8 (3), 216-234. http://dx.doi.org/10.17583/rimcis.2019.4919

Vogels-Broeke, M., Vries, R., \& Nieuwenhuijze, M. (2020). Dimensions in women's experience of the perinatal period. Midwifery, 83, 102602. https://doi.org/10.1016/j.midw.2019.102602

Zanardo, G. L. P., Calderón, M., Nadal A. H. R., \& Habigzang, L. F. (2017). Violência obstétrica no Brasil: uma revisão narrativa. Psicologia \& Sociedade, 29, e155043. https://doi.org/10.1590/18070310/2017v29155043

World Health Organization. (2016). Who recommendations on antenatal care for a positive pregnancy experience.https://www.who.int/reproductivehealth/publications/maternal_perinatal_health/ancpositive-pregnancy-experience/en/

World Health Organization. (2018). Who recommendations: intrapartum care for a positive childbirth experience. https://www.who.int/reproductivehealth/publications/intrapartum-care-guidelines/en/ 